\title{
A Low-Cost PIV System for Undergraduate Fluids Laboratories
}

\section{Aaron Budd, The Citadel \\ Dr. Jason Howison, The Citadel}

Jason Howison is an assistant professor of mechanical engineering at The Citadel. He received his Ph.D. in aerospace engineering from the University of Tennessee. Prior to returning to school, he worked in industry as a solid rocket ballistic analyst. His current research areas include computational fluid dynamics, turbulence modeling, wind turbines, aeroelasticity, and engineering education. 


\title{
A Low-Cost PIV System for Undergraduate Fluids Laboratories
}

\begin{abstract}
Particle image velocimetry (PIV) has become a mature technology used in research and industry alike to quantify flow properties non-intrusively. Unfortunately, the cost remains too high for students to apply the method in most undergraduate fluids laboratories. Moreover, high-powered pulsed lasers normally utilized in PIV systems can be a safety risk in the presence of large groups of undergraduates. In this paper, a low-cost PIV acquisition and analysis system that can be readily implemented in undergraduate fluids laboratories is presented. A low-power, constant laser light source is employed to reduce cost and maintain lab safety. An open source analysis code is also used to minimize cost. In working lab exercises with this system, students will understand the PIV data acquisition process, apply MATLAB to analyze the data, and explain the observed flow features. Details of the system are provided so that others may construct a similar system for use in their laboratory. Sample results for flow over a cylinder inside a water flume are included as a demonstration of the system.
\end{abstract}

\section{Introduction}

Particle image velocimetry (PIV) first appeared in the literature in 1984 [1]. PIV is a nonintrusive measurement technique where particles are injected into a flow field. The particles are illuminated by a laser sheet, and two pictures are taken close together in time. If the distance the particles travel over the time between pictures is determined, the velocity of the particles is determined.

The first PIV systems used film cameras, and it was thought that digital processing was not even possible. The method came into its own in the early 1990s as digital image analysis became a reality [2], [3] and cross-correlation analysis eliminated ambiguity in the flow direction in the data [4]. Up until that point, cameras were not fast enough to acquire two images in close succession. As an alternative, both images were captured on a single exposure. The velocity can still be determined from these images using autocorrelation, but there is directional ambiguity since the software has no way of knowing which particles are from the first or second exposure. Since that time, PIV has grown into a mature experimental method capable of mapping three dimensional velocity fields from a plane of measurement [5] or an entire volume [6].

While increases in digital photography capability have greatly enhanced what is possible with PIV, it has also driven costs up. Similarly, software to analyze images captured on several cameras to produce three-dimensional velocity fields has gotten increasingly expensive. It is not uncommon to see the cost of a commercial PIV system start in six figures. For student laboratory use, this is prohibitively costly. Moreover, this is only a single system that several hundred students may need to use for lab assignments at larger schools.

Most of the components required for a basic PIV system can now be purchased separately for only a few thousand dollars total. There are also several open source computer codes to analyze the images. Following the lead of Ryerson and Schwenk [7], this paper will outline the setup of a cost-effective PIV system suitable for use in undergraduate laboratories. Major components of 
the system will be described and sample results provided. Plans for inclusion into undergraduate courses and expected learning outcomes are also given.

\section{PIV System Description}

A low-cost PIV system can allow students to observe the dynamics of steady and even unsteady flows. Particles with a density similar to the fluid are selected in order to achieve a homogenous distribution within a controlled system. A laser produces the high intensity, collimated light source necessary for image capturing. The laser is transformed into a planar sheet using optical components, which, in turn, illuminates the scattered particles. Each individual frame captured is matched with the successive frame to determine the displacement of particles between frames. The idea is illustrated in Fig. 1.

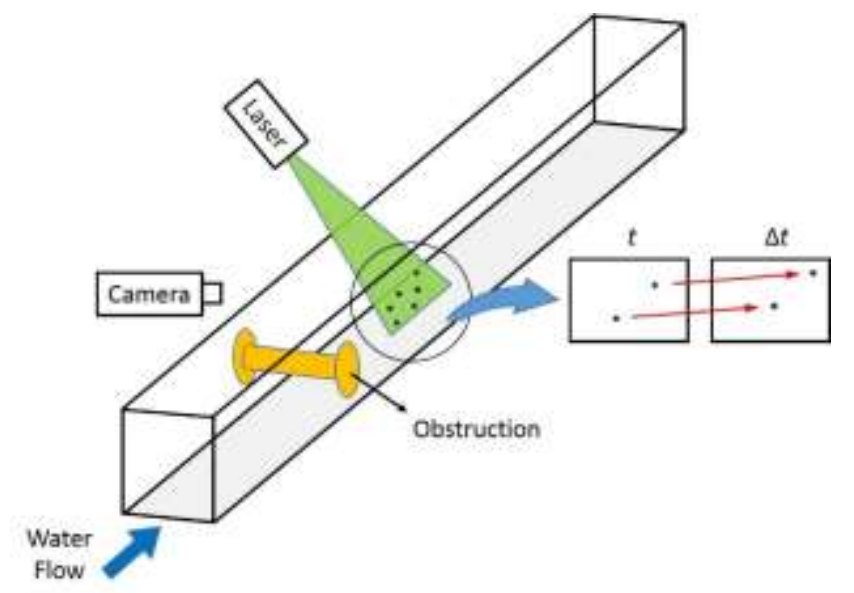

Figure 1: A basic two-dimensional PIV system

Professional systems can start around \$100,000 and use ultra-high speed cameras coupled with synchronized laser pulses to achieve high quality images. The current PIV system costs less than $\$ 2500$, and the limitations mostly occur around the camera's image capturing sensor and resolution. The system components are listed in Table 1. The six megapixel Casio EX-F1 camera is capable of recording $60 \mathrm{fps}$ at maximum resolution $(2825 \times 2124)$, which is sufficient for the expected velocities near $0.2 \mathrm{~m} / \mathrm{s}$. The maximum recording speed of this camera is $1200 \mathrm{fps}$ at $336 \times 96$ resolution. This speed would allow for much greater flow speeds, but the clarity of the images are sacrificed at higher frame rates. The higher frame rate images also contain exceptionally more noise. Unfortunately, the Casio EX-F1 is no longer available for new purchase in the United States, but plenty of similar cameras exist. For example, Ryerson and Schwenk [7] used the Casio EX-FH25, which has since been replaced by the EX-FH100. These cameras are cheaper than the EX-F1 and would work just as well in the present system. Since the high speed function is not high enough resolution for PIV, similar systems only need a camera with 60 fps capability and similar resolution.

The other major system component is the light source. Research level systems use pulsed $\mathrm{Nd}$ :YAG lasers because of their ability to give high-powered, short durations of illumination. For the low speed flows being considered with the current system, it is possible to use a continuous 
light source. If the velocity of the particles is low and the shutter speed is sufficiently high, the particles will not smear in the acquired images. Continuous lasers are relatively inexpensive, smaller, and much safer for groups of undergraduate students to operate. A $150 \mathrm{~mW}$ diodepumped solid-state (DPSS) $532 \mathrm{~nm}$ laser from Laserglow Technologies was used in this work.

The remaining components include light sheet optics, seeding particles, and MATLAB. Most schools have MATLAB academic licenses, but students can also purchase their own student version for $\$ 100$. It is possible to manufacture seeding particles, but in a closed-circuit water flume, a one-time expense of $\$ 149$ is reasonable. The particles lasted several months in the system without noticeable degradation. The optics train will be discussed in the next section, but a couple of spherical and cylindrical lenses and mounting hardware are typical minimum requirements.

Table 1: Equipment and Price List for Low-Cost PIV System

\begin{tabular}{lcc} 
Item & Quantity & Cost \\
\hline *Casio EX-F1 Camera & 1 & $\$ 700$ \\
Laserglow Technologies Low-Cost DPSS Laser & 1 & $\$ 699$ \\
532 nm Wavelength & 1 & $\$ 100$ \\
MATLAB (student license) & 1 & free \\
PIVLab & 1 & $\$ 149$ \\
Cospheric Fluorescent Red Polyethylene & 1 & $\$ 482$ \\
Microspheres 0.995g/cc 10-90um - 10g & 1 & $\$ 156$ \\
Light Sheet Optics & & $\$ 2286$
\end{tabular}

\section{Experimental Facility}

In the experiments, a water flume was utilized that is 2 meters long with a $50 \mathrm{~mm}$ channel width and a max height of $115 \mathrm{~mm}$. It is a standard flume for undergraduate hydrology lab exercises, and it can be seen in Fig. 2a. The height and flow rate of the water can be adjusted using the pump speed and exit flow valve. The volumetric flow rate in the flume can be calculated by measuring the volume of water captured over a period of time, and the average velocity calculated using the cross-sectional area of the water at a given location.

The $1.5 \mathrm{~mm}$ collimated beam generated by the laser passes through a plano-convex cylindrical lens to produce a planar sheet. The focal length of the lens is $f=25 \mathrm{~mm}$, and the height of the sheet diverges $1.5 \mathrm{~mm}$ every $25 \mathrm{~mm}$ after the focal point. The expansion of the sheet is low enough that a second lens is not used to prevent the sheet from diverging. The sheet is reflected off of $50.8 \mathrm{~mm}$ broadband, dielectric mirror before entering the flume.

The fluorescent red seeding particles range in size from 10-90 micrometers and have a density of $0.999 \mathrm{~g} / \mathrm{ml}$. These particles were selected because of their high fluorescence for $532 \mathrm{~nm}$ light. A little trial and error was necessary to get the correct seeding density in the water. Too many particles make the images oversaturated and difficult for the analysis program to track the movement of individual particles between frames. Too few particles create less statistical 
certainty in the area of interrogation. The current system uses one 10 gram vial of particles for approximately 18 gallons of water.

SolidWorks and MakerBot 3D printing machines are available at most universities, and they were used to quickly and cheaply configure mounts and experimental shapes in this experiment. A simple, adjustable mirror mount was printed as well as a cylinder to be inserted in the flume as a test object. Figure $2 \mathrm{~b}$ shows all equipment in place and the laser turned on. The laser is aimed down the flume in the direction of the flow with the camera facing normal to the illuminated plane. The laser is turned on briefly to adjust the mirror angle and camera location. Once the manual focus is adjusted to the plane's location a reference image is taken for calibration.

a)

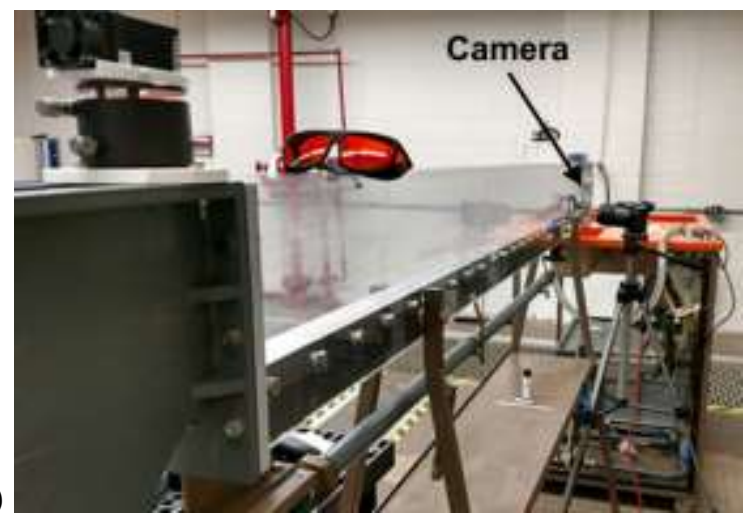

b)

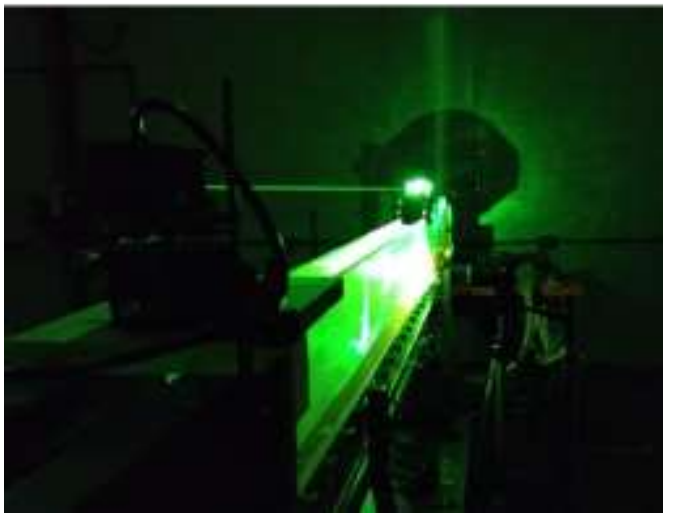

Figure 2: Water flume (a) before experiment begins and (b) with laser turned on.

\section{Data Processing}

PIVLab is an open-source toolbox for MATLAB that processes the images captured and provides quantitative information about the flow. A selected sequence of frames are imported into PIVLlab where preprocessing options such as masking can be done. The program calculates velocity vectors based on the displacement of particles between image pairs, and a vector map is superimposed over the interrogation windows. A calibration image is required to tell the software the relationship between pixels and physical space. Post-processing options such as vector validation can be done to exclude erroneous vectors, and blank spaces can be filled with interpolated vectors if desired. PIVLab will also compute derived quantities such as vorticity from the calculated velocity field.

\section{Results}

To demonstrate the system, the classic case of a cylinder in crossflow was run. A cylinder, $5 \mathrm{~mm}$ in diameter, was placed in the flume. The volumetric flow rate of the flume was set such that the average velocity was approximately $0.2 \mathrm{~m} / \mathrm{s}$. A sample of the raw image file can be seen in Fig. 3a beside a PIVlab, post-processed image pair which produced the vector map in Fig. 4b. Red $x$ 's cover the masked exclusion area while the green arrows fill the interrogation window. Orange arrows in the interrogation windows are interpolated points used to fill gaps where no particle information could be analyzed. Velocities calculated far away from the cylinder are near the approximate value from the volumetric flow rate calculation. 


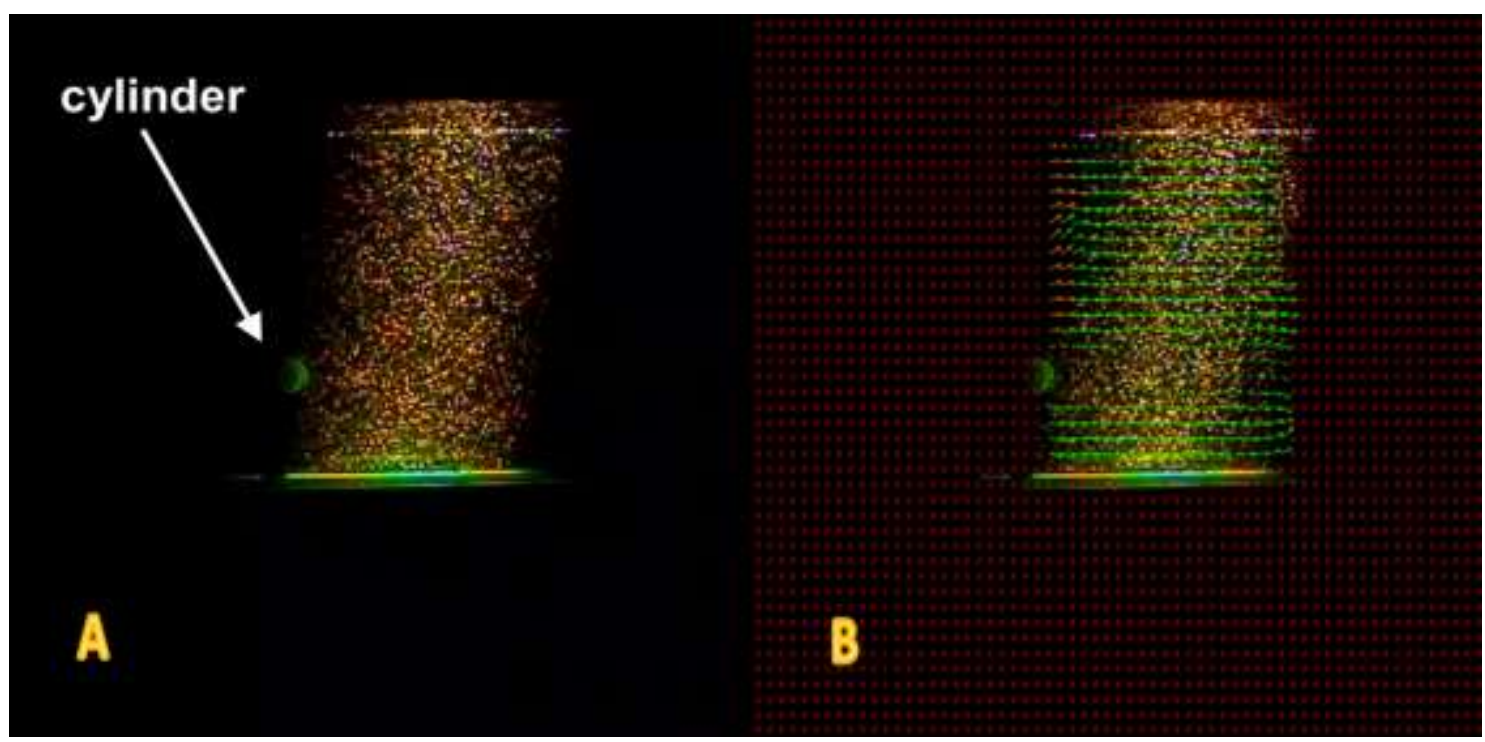

Figure 4: (a) Raw image and (b) post-processed image with vector map

Vortex shedding is a predictable pattern in low Reynolds number flows behind a cylinder. The alternating vortices in the wake are captured by the system and are visually shown using the PIVLab software (Fig. 5). Dark colors indicate a lower velocity and the sinusoidal shape represents the oscillating pattern formed. The vortex locating tool gives a better picture of the vortices formed behind the cylinder. The yellow colors in Fig. 6 indicate the areas where vortices have formed. Red arrows have been inserted in the photo to show the direction of circulation because the tool does not have that capability.

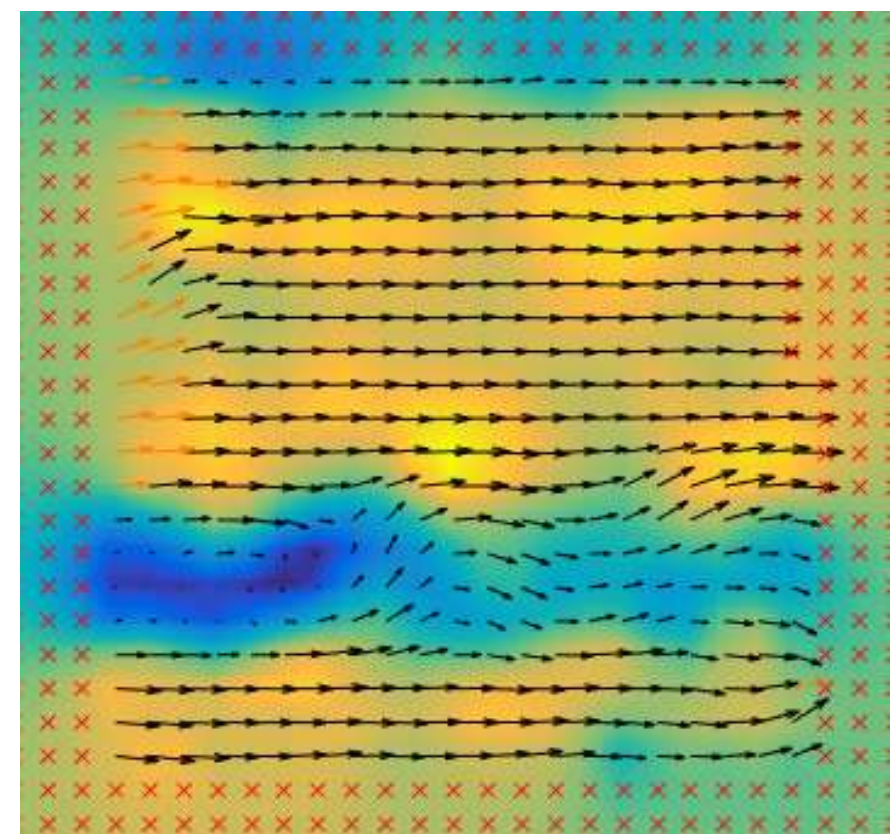

Figure 5: The x-component of velocity. 


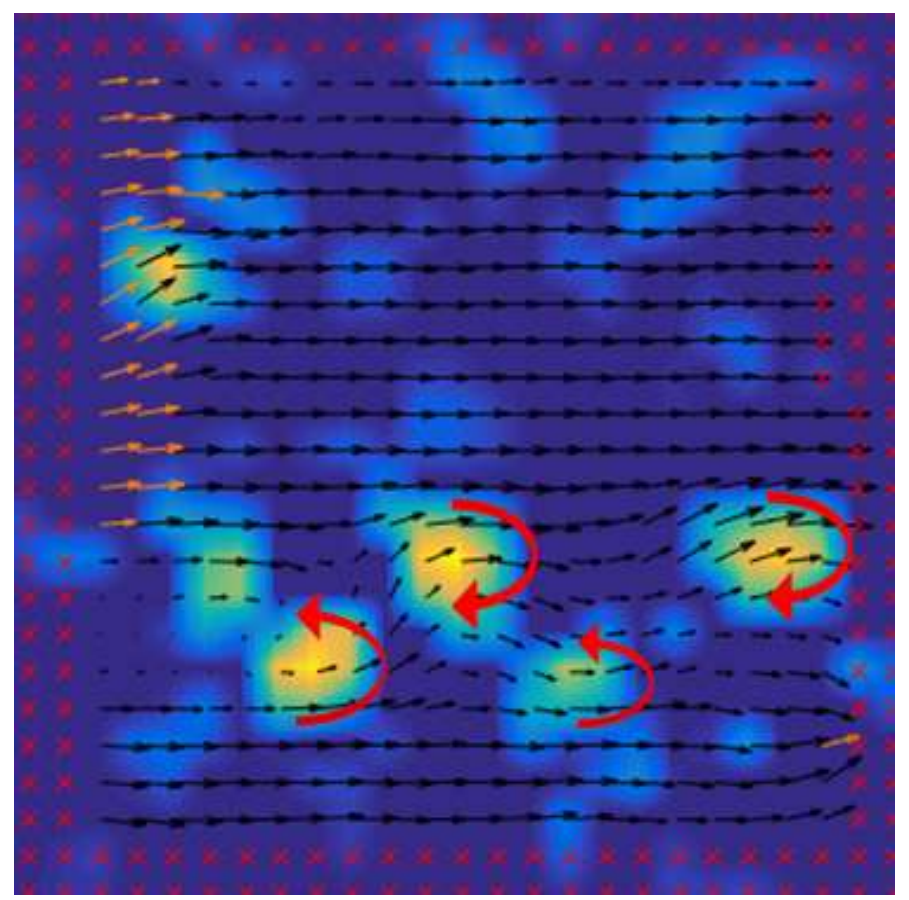

Figure 6: Demonstration of vortex locator tool.

\section{Extension to Fluids Laboratories}

The work presented herein represents an initial effort to bringing PIV experiments into undergraduate courses in thermal/fluids sciences. The long term plan is to implement the system presented on a table-top closed-loop water tunnel. A senior design team is currently designing and constructing the water tunnel. When completed, this will enable PIV measurements to be taken in an ordinary classroom either as a demonstration or lab activity.

In order to successfully complete a lab exercise, students will understand the PIV data acquisition process, apply MATLAB to analyze the data, and explain the observed flow features using information from the textbook and lectures. Each of these learning outcomes can be mapped directly to ABET student outcomes. The PIV data acquisition process applies to outcome (b) an ability to design and conduct experiments, as well as to analyze and interpret data. Using MATLAB to analyze the data applies to outcome $(\mathrm{k})$ an ability to use the techniques, skills, and modern engineering tools necessary for engineering practice. Finally, explaining the features in the flow relates to outcome (a) an ability to apply knowledge of mathematics, science and engineering.

Since this system has yet to be implemented in a course, there is no assessment data available. However, when it is, a typical student report on the lab will be broken down into three parts for assessment. Successful setup of equipment and acquisition of data will map to outcome (b). Successful processing of the image files will map to outcome (k). Finally, students' ability to explain the observed flow phenomena will feed into outcome (a). 


\section{Summary}

A low-cost PIV system has been presented in this paper. This system costs less than $\$ 2500$, which is two orders of magnitude cheaper than research grade systems. At this cost, it is possible to bring the method into undergraduate fluids laboratories so that students can learn and apply the technique. A simple demonstration of the system about a cylinder in crossflow was provided as well as an equipment list. Several learning outcomes can be matched directly to ABET student outcomes.

\section{References}

[1] Adrian, R.J., "Scattering particle characteristics and their effect on pulsed laser measurements of fluid flow: speckle velocimetry vs. particle image velocimetry," Applied Optics, Vol. 23, pp. 1690-1691, 1984. doi:10.1364/AO.23.001690

[2] Willert, C.E. \& Gharib, M., "Digital particle image velocimetry," Experiments in Fluids, Vol. 10, pp. 181-193, 1991. doi:10.1007/BF00190388

[3] Westerweel, J. "Analysis of PIV interrogation with low-pixel resolution," Proc. SPIE 2005, Optical Diagnostics in Fluid and Thermal Flow, December 1993. doi: 10.1117/12.163745

[4] Keane, R.D. \& Adrian, R.J. "Theory of cross-correlation analysis of PIV images," Applied Scientific Research, Vol. 49, pp. 191-215, 1992. doi:10.1007/BF00384623

[5] Guezennec, Y.G., Brodkey, R.S., Trigui, J., Kent, J.C., "Algorithms for fully automated three-dimensional particle tracking velocimetry," Experiments in Fluids, Vol. 17, pp. 209-219, 1994. doi: 10.1007/BF00203039

[6] Elsinga, G.E., Scarano, F., Wieneke, B. et al., "Tomographic particle image velocimetry," Experiments in Fluids, Vol. 41, pp. 933-947, 2006. doi:10.1007/s00348-006-0212-Z

[7] Ryerson, W.G. \& Schwenk, K., “A simple, inexpensive system for digital particle image velocimetry (dpiv) in biomechanics,” J. Exp. Zool. Vol. 317, pp. 127-140, 2011. 